\title{
Expression profile of RhoGDI2 in lung cancers and role of RhoGDI 2 in lung cancer metastasis
}

\author{
HUIYAN NIU ${ }^{1}$, HUI LI ${ }^{1}, \mathrm{CAN}^{\mathrm{X}} \mathrm{U}^{2}$ and PING HE${ }^{1}$ \\ Departments of ${ }^{1}$ Geriatrics, ${ }^{2}$ Pathology, Shengjing Hospital, China Medical University, Shenyang 110004, P.R. China
}

Received March 22, 2010; Accepted April 30, 2010

DOI: $10.3892 /$ or_00000880

\begin{abstract}
Rho GDP dissociation inhibitors (RhoGDIs) are important regulators of the GTP hydrolase activity and biological functions of Rho GTPases. RhoGDI2 has been shown to be a metastasis suppressor in bladder cancer and several other cancers. However, the underlying mechanism, effector targets, and the cognate biological functions of RhoGDI2 are not fully understood. To investigate the possible role of RhoGDI2 in lung cancer tumorigenesis and metastasis, the expression pattern of RhoGDI2 in various lung cancer tissue samples and lung cancer-derived cell lines were profiled at both mRNA and protein levels. Furthermore, possible interplay between PI3K/Akt/mTOR pathway activation/inhibition and RhoGDI2 signalling is examined in lung cancer-related cell lines. Our results suggest that RhoGDI2 is likely to be involved in lung tumor malignancy and metastasis.
\end{abstract}

\section{Introduction}

Lung cancer is both the leading cause of cancer-related deaths in men and one of the most common forms of cancer in women, killing more than one million people annually worldwide (http://www.who.int/mediacentre/factsheets/ fs297/en/). Clinically, lung cancer remains a disease with dismal prognosis. This has been attributed mainly to the low cost-effectiveness of early detection methods and the lack of successful therapeutic options for the highly metastatic forms of lung cancer (1).

Metastasis, recognized as one of the hallmarks of malignancy, is a complex, multi-step process in which cancer cells migrate from the original tumor site to other locations in the body. During metastasis, tumor cells usually exhibit some

Correspondence to: Dr Ping He, Department of Geriatrics, Shengjing Hospital, China Medical University, Shenyang 110004, P.R. China

E-mail: hep@sj-hospital.org

Key words: lung cancer, Rho GDP dissociation inhibitor 2, PI3K/ $\mathrm{Akt} / \mathrm{mTOR}$ signalling pathway, invasion, metastasis characteristics that normal and non-metastatic cancer cells lack. On one hand, these acquired characteristics enhance the invasive and migrant nature of metastatic tumor cells. On the other hand, the expression of certain molecules in normal and benign cancer cells is found to suppress the metastasis process and these molecules are often lost in metastasizing cancer cells, whereas some molecules are found specifically up-regulated in metastatic tumors.

An antithetical group of genes encode molecules that specifically regulate the spread of cancer cells but not the tumorigenicity by targeting multiple steps of the metastatic process (2). One of these molecules, RhoGDI2, was initially identified as metastasis suppressor in bladder cancer and later found to be lost in many metastatic human tumors (3).

RhoGDI2 belongs to a small family of proteins that also include RhoGDI1 and RhoGDI3. RhoGDI2 is primarily expressed in hematopoietic and endothelial cells (4) and is also found differentially expressed in some human cancers. For example, RhoGDI2 suppresses invasion and metastasis in bladder cancer $(3,5)$ and is a positive prognostic factor $(6)$. Moreover, RhoGDI2 is selectively down-regulated in Hodgkin lymphoma cells compared with non-Hodgkin lymphoma (7). In contrast, RhoGDI 2 is thought to be involved in the growth and invasive behavior in ovarian, breast and gastric cancers (8-10). Thus, the actual roles of RhoGDI2 protein in tumor progression remain ununified, suggesting that different regulatory pathways may be involved in different tumors.

One of the key functions of Rho GTPase proteins is to remodel cytoskeleton components thus influence cell mobility. Therefore, it is not surprising that regulation of Rho family proteins can have profound impact on metastasis, a process that involves cancer cells migrating from one location to another and invading the destination tissue/organ. While RhoGDI2 was reported to be involved in the regulation of metastasis of several cancer types, the downstream signalling events have not been fully delineated. In bladder, endothelin and neuromedin were identified to be down-regulated by RhoGDI2 through expression profiling $(11,12)$, whereas Rac-1 was found to be bound and activated by RhoGDI2 (13). Interestingly, Src kinase seems to phosphorylate RhoGDI2 at Tyr153, which stimulates the metastasis suppressor activity of RhoGDI2 (14).

Parallel to Rho proteins, there are other signalling pathways that also regulate cytoskeleton dynamics in the cell, e.g., the Akt signalling pathway. Akt/protein kinase B is a major downstream effector of growth factor receptor tyrosine kinases 
that signal via phosphatidylinositol-3 kinase (PI3K) (15). Receptor-mediated activation of PI3K or loss of expression of phosphatase PTEN, one of the most frequently mutated tumor suppressors, often results in elevated Akt activity. The PI3K/Akt pathway plays a major role in several key cellular processes, including cell survival and apoptosis, glucose metabolism, cell cycle progression, and gene expression (16). Furthermore, PI3K/Akt pathway is one of the major upregulated signalling events during the development of many cancers.

Herein, we explored possible roles of RhoGDI2 in lung cancer, and probed a potential interplay between RhoGDI2 expression and Akt signalling pathway.

\section{Materials and methods}

Clinical samples and cell lines. Paraffin-embedded specimens (112) were obtained from the Shengjing Hospital of China Medical University and 20 frozen specimens were obtained from Liaoning Provincial Tumor Hospital; all with consent from patients. Fresh cancerous specimens and corresponding normal tissue samples were plunged into liquid nitrogen immediately after resection and stored at $-80^{\circ} \mathrm{C}$ until the extraction of RNA and protein.

Lung cancer cell lines A549, SPC-A-1, 95D and NCI-H446 were purchased from Shanghai Institute of Biochemistry and Cell Biology, Chinese Academy of Sciences. HeLa cell line is a gift of the Department of Cell Biology, China Medical University.

Reagents and antibodies. Recombinant human HGF (rhHGF) was purchased from R\&D Systems (Minneapolis, MN). LY294002 and rapamycin were purchased from Sigma Chemical (Sigma, St. Louis, MO). Anti-RhoGDI2 was purchased from Abcam Inc. (Cambridge, MA). Anti-GAPDH were purchased from Santa Cruz Biotechnology (Santa Cruz, CA).

Immunohistochemisty. Immunostaining was performed using the immunohistochemical SP (streptavidin-peroxidase) method. Sections were deparaffinized in xylene, and rehydrated in gradients of alcohol and water. Tissues were immunohistochemically stained with diluted primary antibody against RhoGDI2 (1:100) overnight at $4^{\circ} \mathrm{C}$ using a Histostain-Plus kit (Zymed, Invitrogen). Five random non-overlapping views were chosen per slide; at least 100 cells were observed and examined per view at x400 magnification. Labelling scores were determined by the percentage of positive cells per slide. Results were compared using the Shimizu et al standard (17).

$R T-P C R$. Total RNA was extracted using TRIzol reagent (Invitrogen) according to the manufacturer's instructions. RTPCR was performed using the RNA PCR kit (AMV) version 3.0 (Takara) following the manufacturer's instructions. RT-PCR products were analyzed on $1 \%$ agarose gels. The sequences of the PCR primers are: RhoGDI2-upstream (5'-ATCGAATTC atgactgaaaaagccccagag-3'), RhoGDI2-downstream (5'-ATT CTCGAGtcattctgtccactccttc-3'), GAPDH-upstream (5'-GAA GGTGAAGGTCGGAGTC-3'), and GAPDH-downstream (5'-GAAGATGGTGATGGGATTTC-3').
Cell culture. A549, SPC-A-1, 95D and NCI-H446 cells were cultured in RPMI-1640 medium containing $10 \%$ fetal calf serum, $100 \mathrm{IU} / \mathrm{ml}$ penicillin and $100 \mu \mathrm{g} / \mathrm{ml}$ streptomycin. The cells were grown in a humidified incubator at $37^{\circ} \mathrm{C}$ and $5 \% \mathrm{CO}_{2}$ air atmosphere. Cells were grown on sterilized tissue culture petri dishes and passaged once every 2-3 days. Various treatment of cells included the addition of rhHGF (10 and $30 \mathrm{ng} / \mathrm{ml}), \operatorname{LY} 294002(5,20,50 \mu \mathrm{mol} / \mathrm{l})$, or rapamycin $(10,20$, $40 \mathrm{nM}$ ), or the combination of LY294002 and rapamycin (LY $5 \mu \mathrm{mol} / \mathrm{l}+\mathrm{R} 10 \mathrm{nM}$, LY $10 \mu \mathrm{mol} / \mathrm{l}+\mathrm{R} 10 \mathrm{nM})$.

Immunofluorescence. Coverslides with grown cells were washed with PBS and fixed with $4 \%$ paraformaldehyde at room temperature for $20 \mathrm{~min}$ before being soaked in antiRhoGDI2 antibody $(1: 100)$ in the wet box at $4^{\circ} \mathrm{C}$ overnight. After washing away unbound primary antibodies, FITClabeled goat anti-rabbit secondary antibody was added in the dark room and incubated at $37^{\circ} \mathrm{C}$ for $30 \mathrm{~min}$. After washing off the unbound secondary antibodies, the results were observed via fluorescence microscopy.

Western blotting. Western blotting was performed using standard techniques as previously described. Briefly, cells were washed twice with PBS buffer and lysed in RIPA lysis buffer (50 mM Tris-Cl pH 7.4, $150 \mathrm{mM} \mathrm{NaCl}, 0.5 \%$ sodium deoxycholate, $1 \%$ NP-40, 0.1\% SDS, 1 mM EDTA, $100 \mathrm{mM}$ $\mathrm{NaF}, 1 \mathrm{mM} \mathrm{Na} \mathrm{VO}_{4}, 1 \mathrm{mM}$ PMSF, and $2 \mu \mathrm{g} / \mathrm{ml}$ aprotinin) on ice. Total proteins $(50 \mu \mathrm{g})$ were subjected to sodium dodecyl sulfate-polyacrylamide gel electrophoresis (SDS-PAGE) and transferred to polyvinylidene difluoride membranes. Membranes were blocked with 5\% nonfat milk in TBST $(10 \mathrm{mM}$

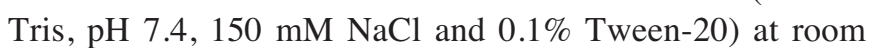
temperature for $2 \mathrm{~h}$ and incubated with the indicated primary antibodies at $4{ }^{\circ} \mathrm{C}$ overnight with gentle rocking. After washing with TBST, the membrane was reacted with the appropriate horseradish peroxidase (HRP)-conjugated secondary antibodies for $1 \mathrm{~h}$ at room temperature. After extensive washing with TBST, proteins were visualized by the enhanced chemiluminescence (ECL) detection kit in accordance with the manufacturer's recommendations.

Monolayer wound healing assay. 95D cells were incubated in 6 -well plates to reach full confluence in monolayer. The monolayers were then disrupted by scraping with a pipette tip, incubated with or without drugs, and observed every $2 \mathrm{~h}$ until the wound was completely closed.

Matrigel invasion assay. Diluted matrigel was added to the upper chambers of a 24-well transwell. In the upper chambers, $200 \mu \mathrm{l}$ cells were grown in serum-free medium on $8 \mu \mathrm{m}$ porous polycarbonate membranes, which were coated with matrigel basement membrane matrix (BD Biosciences, Bedford, MA). The lower chambers were filled with RPMI1640 medium containing $20 \%$ fetal calf serum. After incubation for $24 \mathrm{~h}$ at $37^{\circ} \mathrm{C}$ in a humid atmosphere of $5 \%$ $\mathrm{CO}_{2}$, the cells that had migrated through the pores were fixed with ethanol for $30 \mathrm{~min}$ and stained with crystal violet. The number of cells was then counted visually under microscope using an averaged reading obtained in five different viewing fields. 
Table I. Statistical analysis of the expression level of RhoGDI2 protein in relation to tumor characteristics in clinical samples of lung cancer.

\begin{tabular}{|c|c|c|c|c|c|}
\hline & \multicolumn{3}{|c|}{ Case no. } & \multirow[b]{2}{*}{$\chi^{2}$} & \multirow[b]{2}{*}{$\mathrm{P}$} \\
\hline & Total & - & + & & \\
\hline \multicolumn{6}{|l|}{ Gender } \\
\hline Male & 76 & 46 & 30 & 0.393 & 0.531 \\
\hline Female & 36 & 24 & 12 & & \\
\hline \multicolumn{6}{|l|}{ Age } \\
\hline$\leq 60$ & 59 & 39 & 20 & 0.690 & 0.406 \\
\hline$>60$ & 53 & 31 & 22 & & \\
\hline \multicolumn{6}{|l|}{ Cancer type } \\
\hline Squamous cell lung cancer & 59 & 34 & 25 & 1.263 & 0.261 \\
\hline Adenocarcinoma & 53 & 36 & 17 & & \\
\hline \multicolumn{6}{|l|}{ Cell differentiation level } \\
\hline High & 20 & 8 & 12 & 16.123 & $<0.01$ \\
\hline Medium & 73 & 43 & 30 & & \\
\hline Low & 19 & 19 & 0 & & \\
\hline \multicolumn{6}{|l|}{ TNM grade } \\
\hline I-II & 89 & 48 & 41 & 13.573 & $<0.01$ \\
\hline III-IV & 23 & 22 & 1 & & \\
\hline \multicolumn{6}{|l|}{ Spread to lymph nodes } \\
\hline Yes & 55 & 51 & 4 & 42.130 & $<0.01$ \\
\hline No & 57 & 19 & 38 & & \\
\hline
\end{tabular}

siRNA knock-down of RhoGDI2 expression in lung cancer cells. Synthetic siRNA (sequences GCAGCUUUGACCA CUCUAA and CCACCAUCACAGAGUGUCU) targeting RhoGDI 2 coding region were transfected into A549 cells using the Lipofectamine 2000 kit (Invitrogen) according to manufacturer's instructions. Cells were allowed to recover for 24 or $48 \mathrm{~h}$ prior to being subjected to fluorescence-activated cell sorting (FACS) analysis. Each experiment setting was repeated in triplicates and the percentage of cells in different phases of the cell cycle was averaged and recorded.

Statistical analysis. All statistical calculations were performed using SPSS13.0 software. P>0.05 were considered statistically significant.

\section{Results}

Expression level of RhoGDI2 is inversely correlated with the malignancy grade of the lung cancer tissues studied. We first sought to analyze the expression level of RhoGDI2 in various clinical lung cancer specimens via immunohistostaining. A strong correlation is observed between the downregulation of RhoGDI2 expression and the higher malignancy grade (III-IV) of lung cancer $(\mathrm{p}<0.01$, see Table I). Accordingly, lower cell differentiation level and greater lymph node metastasis propensity are also associated with lower level of RhoGDI2 expression ( $\mathrm{p}<0.01$, Table I). Other factors, such as age and gender, do not seem to be linked with RhoGDI2 expression ( $\mathrm{p}>0.05$, Table I). Interestingly, RhoGDI2 expression seems to be stronger in squamous carcinoma than in adenocarcinoma specimens. However, the result of statistical analysis prevents us from assigning any meaningful significance to this finding $(\mathrm{p}=0.261$, Table I). A representative comparison in RhoGDI 2 expression between cancerous and paracancerous lung tissues is shown in Fig. 1.

We were interested in finding out whether the downregulation of RhoGDI2 expression occurs at mRNA level as well. For this purpose, total RNAs were extracted from lung cancer specimen and normal lung tissue of the same patient, respectively. RT-PCR results indicate that RhoGDI2 mRNA level is significantly lower in lung cancer tissue than that in normal tissue. A representative gel of the RT-PCR products obtained from clinical specimens is shown in Fig. 2A. Whether the low level of RhoGDI2 mRNA present in lung cancer cells is a result of down-regulated transcription activity or of reduced mRNA processing/stability remains unclear.

Expression and intracellular localization of RhoGDI2 in lung cancer-derived cell lines. We next examined the expression level of RhoGDI2 in lung cancer-derived cell lines using RTPCR and Western blots. While RhoGDI2 is weakly expressed in A549 and NCI-H446, the expression levels in 95D and SPC-A-1 seem to be 2- to 3-fold higher than those observed 

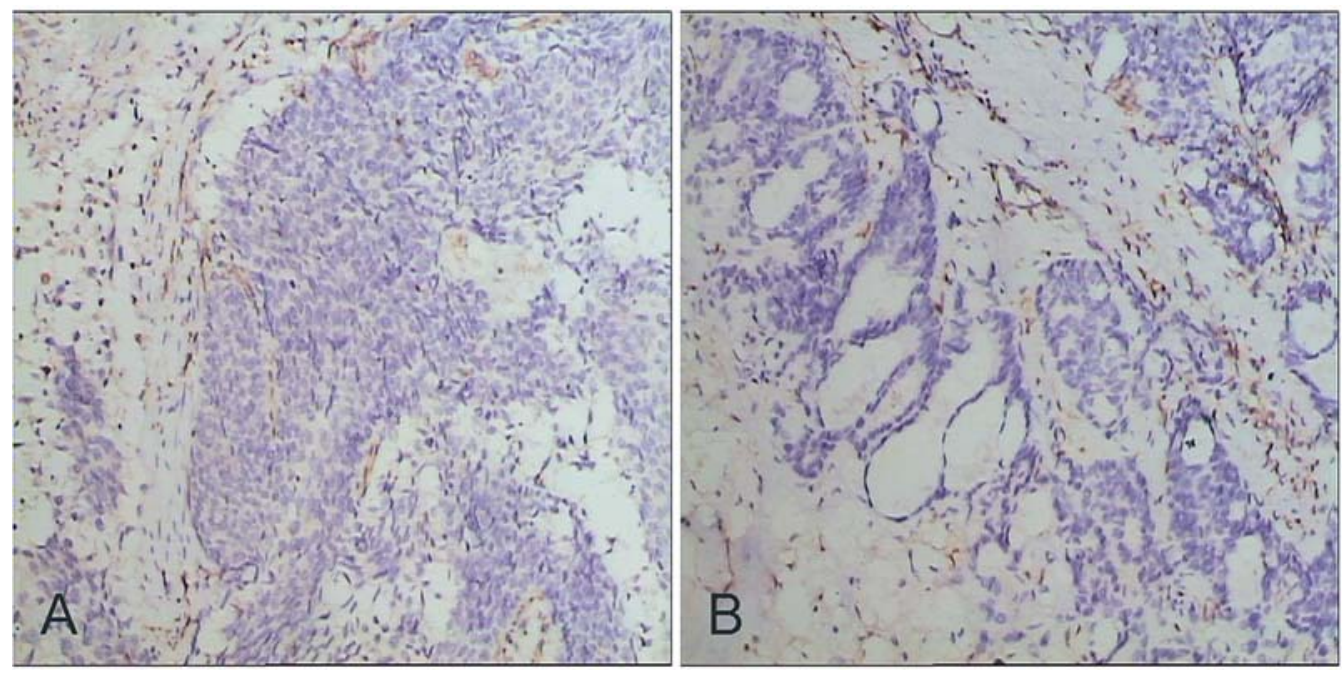

Figure 1. Expression of RhoGDI2 is down-regulated in both squamous lung cell carcinoma and adenocarcinoma. (A) Squamous lung cell carcinoma; (B) adenocarcinoma.

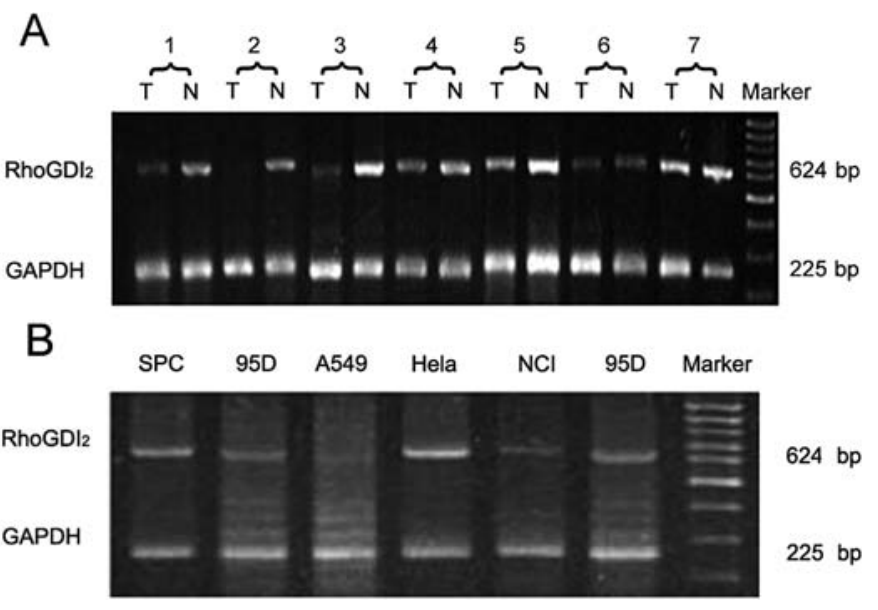

Figure 2. mRNA levels of RhoGDI2 are down-regulated in lung cancer cells compared to normal tissue as detected by RT-PCR. RT-PCR was performed on total RNA extracted from (A) clinical specimens and (B) cultured cell lines. $\mathrm{T}$, tumor site extraction; $\mathrm{N}$, normal tissue near the tumor site.

in the former cell lines (Figs. 2B and 3). To investigate whether lung cancer-derived cell lines express RhoGDI2 in its cognate intracellular compartment, we performed immunofluorescence microscopy on cultured A549 cells. We found that RhoGDI2 is localized primarily in the cytoplasm of A549 cells (data not shown), which is in accordance to its known regulatory functions.

Potential relationship between RhoGDI2 and PI3K/Akt/ mTOR signalling pathway. Given its prominent role in the oncogenesis and development of many lung cancers, the PI3K/ Akt/mTOR pathway was chosen as a potential signalling event that cross-talks with RhoGDI 2 in lung cancer cells. Recombinant human hepatocyte growth factor (rhHGF) is known to promote the growth, motility and invasive ability of $95 \mathrm{D}$ cells through the activation of Akt pathway, whereas

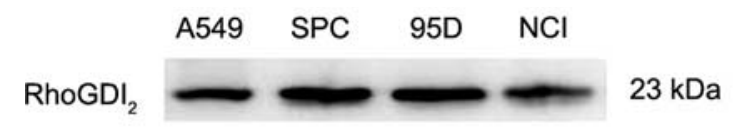

GAPDH $38 \mathrm{kDa}$

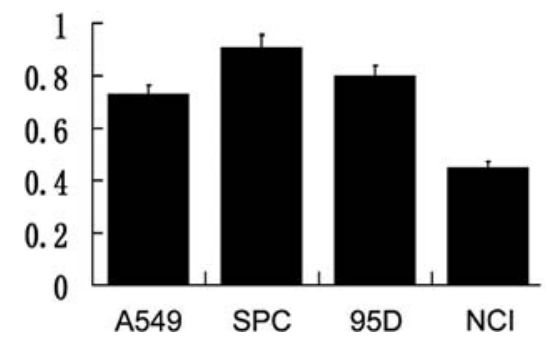

Figure 3. Expression of RhoGDI2 in four lung cancer-derived cell lines. The expression of RhoGDI2 in A549, SPC-A-1, 95D, and NCI-H446 was examined via Western blotting and quantified using endogenous GAPDH as reference.

LY294002 and rapamycin negatively affect these attributes by inhibiting PI3K activity and mTOR pathway, respectively.

In serum-free culture of $95 \mathrm{D}$ cells, the expression of rhoGDI2 is higher than that in $95 \mathrm{D}$ cells cultured in the presence of $10 \%$ fetal bovine serum. rhHGF treatment (10 ng/ $\mathrm{ml}$ ) did not affect the expression of RhoGDI2 compared to that in 95D cell culture in $10 \% \mathrm{FBS}$, whereas $30 \mathrm{ng} / \mathrm{ml} \mathrm{rhHGF}$ significantly reduced it (Fig. 4). This suggests that there may exist an antagonistic relationship between the PI3K/Akt/ mTOR pathway and RhoGDI2 expression in lung cancer cells. This position is further supported by the observation that treatment of 95D cells with LY294002 increased RhoGDI2 expression in a dose-dependent manner (Fig. 5). Moreover, low dosages of rapamycin treatment (10 and $20 \mathrm{nM}$ ) enhanced RhoGDI2 expression, whereas higher dosage of rapamycin at $40 \mathrm{nM}$ actually reduced the level of RhoGDI2, probably because of significant cell deaths (data not shown) or other 

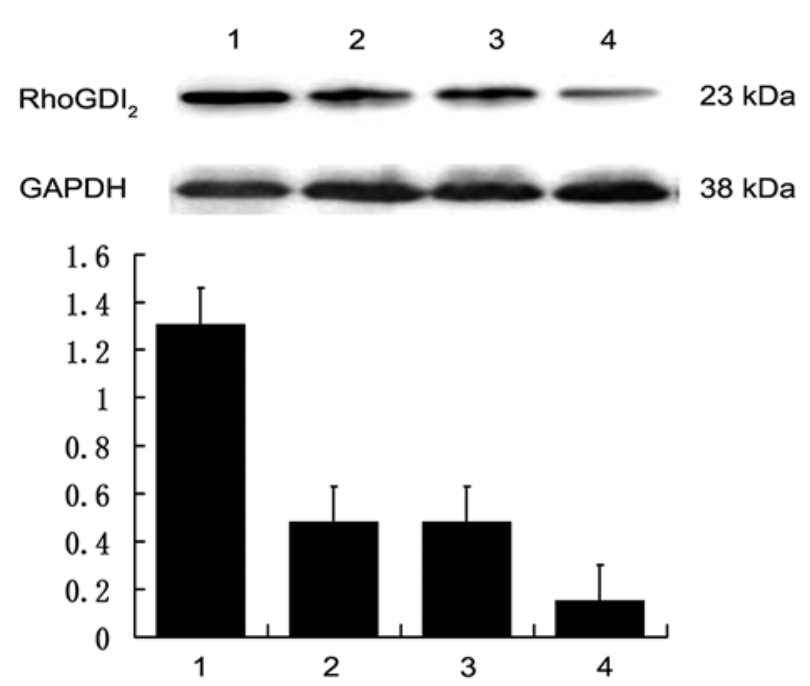

Figure 4. Effect on RhoGDI2 expression in 95D treated with PI3K/ Akt/mTOR agonist. The expression levels of RhoGDI2 as examined via Western blotting and charted results below. 1, Serum-free culture; 2, $10 \%$ FBS supplemented; 3, $10 \mathrm{ng} / \mathrm{ml} \mathrm{rhHGF}$ supplemented; 4, $30 \mathrm{ng} / \mathrm{ml} \mathrm{rhHGF}$ supplemented.

signalling pathways that had become activated at this concentration of rapamycin (see Discussion). Finally, combined treatment of LY294002 and rapamycin additively increased RhoGDI2 expression, implying that the target molecules of these inhibitors each contribute via a different path to the down-regulation of RhoGDI2 in lung cancer cells.

Expression of RhoGDI2 does not positively contribute to cancer cell growth in lung cancer-derived cell lines. Since elevated RhoGDI2 expression was found in breast cancer cell lines and positively linked to breast cancer cell invasion $(10,18)$, we were interested in checking whether RhoGDI2 expression is required for the growth of lung cancer-derived cells. When endogenous RhoGDI2 expression was knocked down by transfecting synthetic RhoGDI2-specific siRNAs into A549 cells, the distribution of S-phase vs. G2-phase cells did not change significantly from those of mock-transfected cells as analyzed by FACS (data not shown). Furthermore, RhoGDI2 knock-down cells did not exhibit higher degree of cell death either in the presence or absence of rapamycin or LY294002. These results indicate that RhoGDI2 does not positively contribute to the growth and survival in lung cancer cells in vitro.

RhoGDI2 is involved in cell mobility and invasion of lung cancer-derived cell lines. We were also interested in finding whether RhoGDI2 knock-down cells exhibited elevated activities in cell invasion $(30 \%$ more than mock-transfected A549 cells). In addition, the siRNA-treated cells showed a marked shortening of time required for wound healing compared to native or mock-transfected A549 cells. These results suggest that RhoGDI2 is a candidate metastasis suppressor in lung cancer. In agreement with this, the treatment of 95D cells with rhHGF enhanced cell invasion and wound healing, whereas the addition of LY294002 and/or rapamycin inhibited these activities (Fig. 6).

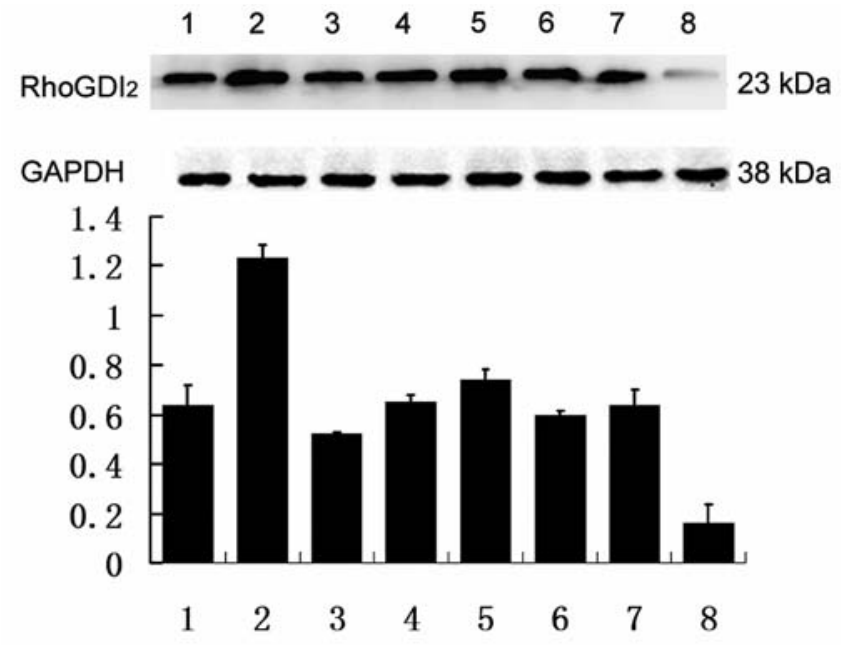

Figure 5. Effects on RhoGDI2 expression in 95D treated with PI3K/ Akt/mTOR antagonist. The expression levels of RhoGDI2 as examined via Western blotting and charted results below. 1, LY294002 (LY) $5 \mu \mathrm{mol} / 1+$ rapamycin (R) $10 \mathrm{nM}$; 2, LY $10 \mu \mathrm{mol} / 1+\mathrm{R} 10 \mathrm{nM}$; 3, LY $5 \mu \mathrm{mol} / 1$; 4, LY $20 \mu \mathrm{mol} / 1$; 5, LY $50 \mu \mathrm{mol} / 1$; 6, R $10 \mathrm{nM}$; 7, R $20 \mathrm{nM} ; 18$, R $40 \mathrm{nM}$.

\section{Discussion}

RhoGDI2 is known to be a metastasis suppressor in bladder cancer. In sharp contrast, RhoGDI 2 promotes cell invasion in breast, ovarian and gastric cancers. However, its role in the oncogenesis, development and metastasis in lung cancer has not been systematically examined so far. In the present study, we aimed to address the following questions: 1) Is RhoGDI2 involved in the oncogenesis, development and metastasis of lung cancer? 2) What are the signalling events involving RhoGDI2 in lung cancer? The expression profile of RhoGDI2 in clinical specimens showed a strong correlation of RhoGDI2 down-regulation and higher malignancy grade of the tumor. This suggests that RhoGDI2 may be involved the development of lung cancer. Expression of RhoGDI2 is also inversely correlated with degree of cell differentiation and propensity of lymph node metastasis in tumor specimens, further suggesting that RhoGDI2 may be involved in lung cancer metastasis. When we examined expression of RhoGDI2 in cultured lung cancer-derived cells, we found that RhoGDI2 expresses at varying levels from cell line to cell line. On one hand, this phenomenon is similar to the varying levels of RhoGDI2 expression detected in clinical specimens. On the other hand, since all four cell lines examined so far showed at least some degree of RhoGDI2 expression, we posit that the anti-metastatic signalling events downstream of RhoGDI2 could have been countered in these cells via some unknown yet reversible mechanisms.

We next focused our study on examining possible signalling events that may involve RhoGDI2 in the progression of lung cancers. Akt signalling pathway is often found activated in most lung cancers. Up-regulation of Akt signalling is positively associated with the malignancy of lung cancers: high level of phosphorylated Akt was detected in excised non-small cell lung cancer (NSCLC) tumor tissue via immunohistochemical method (19). Furthermore, activation of Akt signalling is reported in bronchioloalveolar carcinoma but 

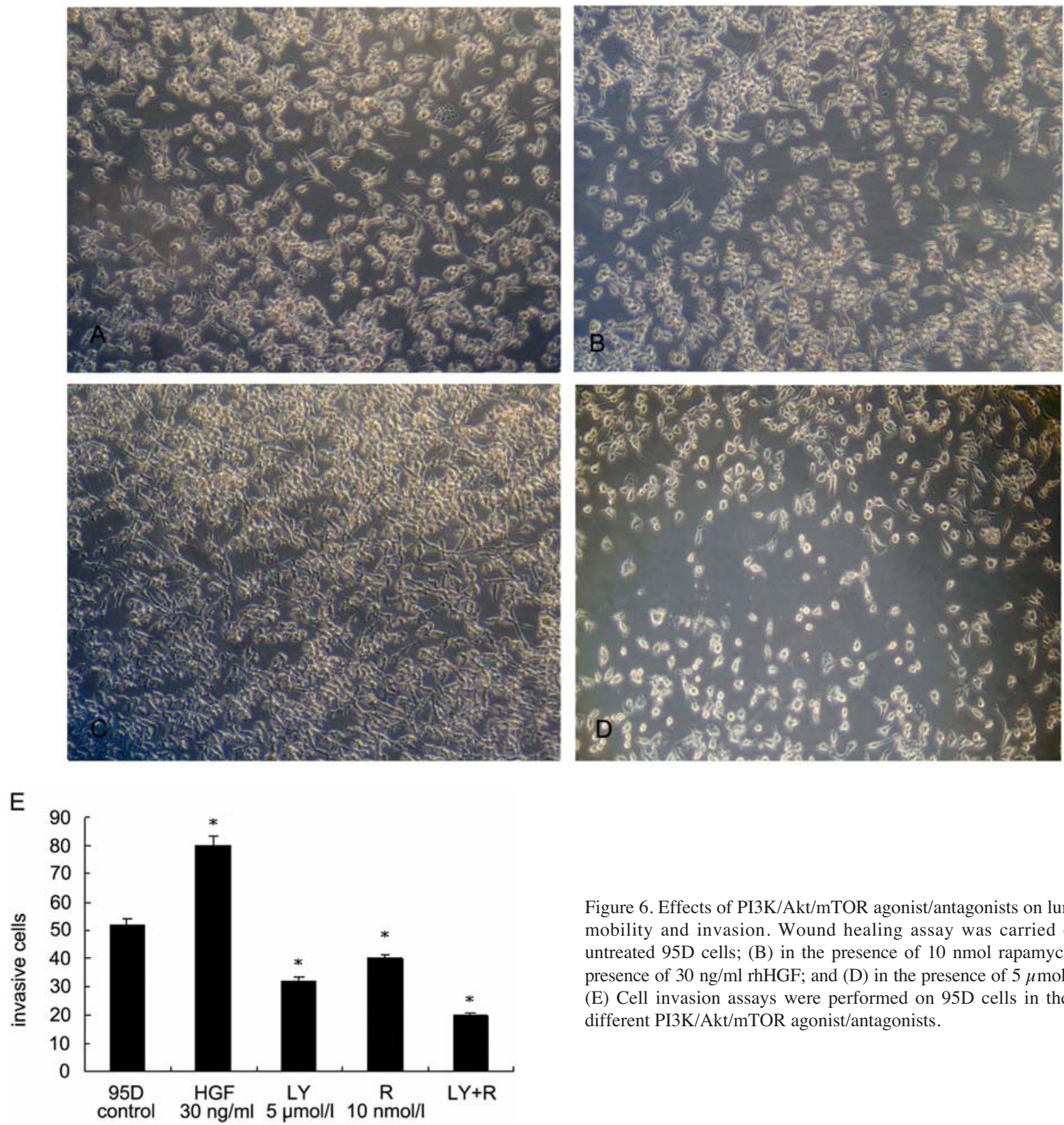

not the corresponding normal cells, suggesting that Akt may play a role in cancer progression to malignancy (20). We were therefore very interested in finding out whether RhoGDI2 signalling events cross-talk with the PI3K/Akt/mTOR pathways. Using specific agonist/antagonists of the PI3K/ Akt/mTOR pathways, we were able to show that the expression of RhoGDI2 is inversely correlated to the level of activity of the Akt/mTOR pathway. Inhibition of PI3K or mTOR alone leads to elevated RhoGDI2 expression and negative impact on cell mobility and invasion in vitro, with the combined inactivation of PI3K and mTOR achieving the best anti-metastasis effects. This suggests that for lung cancer patients with functional RhoGDI2 gene, it may be possible to reactivate RhoGDI2 expression thus inhibit metastasis and cancer progression.

Interestingly, our results with the double inhibition of mTOR and PI3K echoes the recent finding that dual inhibition of parallel signalling pathways in lung cancer such as those via the activation of PI3K and MAPK can prevent undesired

Figure 6. Effects of PI3K/Akt/mTOR agonist/antagonists on lung cancer cell mobility and invasion. Wound healing assay was carried out with (A) untreated 95D cells; (B) in the presence of $10 \mathrm{nmol}$ rapamycin; (C) in the presence of $30 \mathrm{ng} / \mathrm{ml} \mathrm{rhHGF}$; and (D) in the presence of $5 \mu \mathrm{mol} / 1 \mathrm{LY} 294002$. (E) Cell invasion assays were performed on 95D cells in the presence of different PI3K/Akt/mTOR agonist/antagonists.

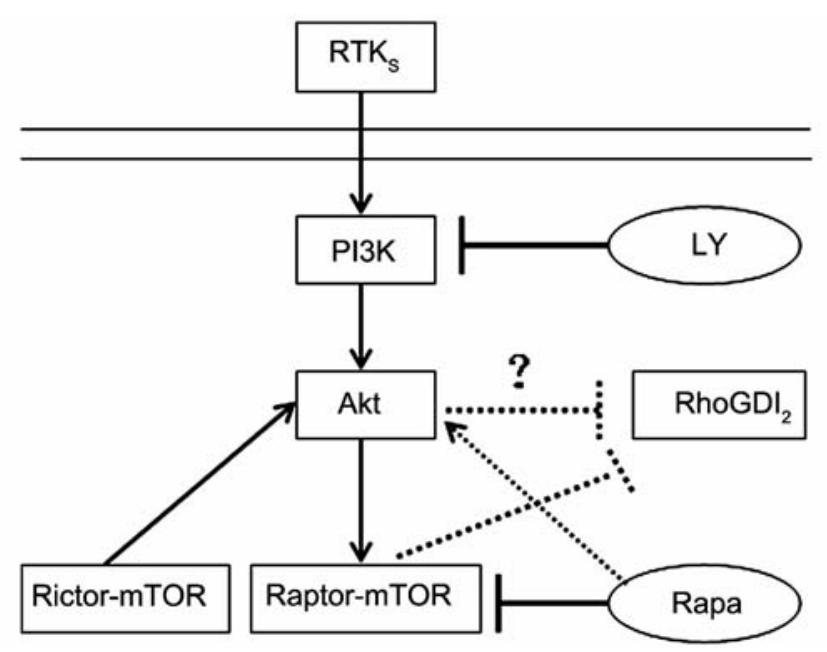

Figure 7. The possible interactions between RhoGDI2 and PI3K/Akt/mTOR pathway. Arrows indicate stimulation, whereas T-lines indicate inhibition. Dotted lines are hypothetical interactions proposed in text. 
reciprocal pathway activation induced by inhibitor-mediated release of negative feedback loops (21). These authors further proposed that the combined inhibition of PI3K and MAPK pathways may provide much better therapy against tumors as parallel signalling pathways are blocked simultaneously. In a similar thread of thought, we think that there might be a clinical benefit in applying combinatorial treatments targeting different components of the PI3K/Akt/mTOR signalling pathways in the treatment of lung cancer.

Based on our data, we propose a potential interaction map between the known components of PI3K/Akt/mTOR pathway and RhoGDI2 in lung cancer metastasis (Fig. 7). In this hypothetical model, both mTOR and Akt can independently antagonize the metastasis suppressor activity of RhoGDI2 by down-regulating its expression. This explains the observation that the dual inhibition by LY294002 and rapamycin led to an additive enhancement on RhoGDI2 expression. Since rapamycin is active on Raptor-mTOR but not Rictor-mTOR complex, higher concentration of rapamycin may shift the intracellular balance from Raptor-mTOR to Rictor-mTOR, which activates Akt by phosphorylating Ser473 in Akt (22). Furthermore, the release of feedback loop inhibition by mTOR inhibitors in cancer cells can lead to upstream receptor tyrosine kinase activation, which again leads to activation of Akt (23). Either or both of these mechanisms could be the reason underlying our observation that RhoGDI2 expression was lower when higher dosage of rapamycin $(40 \mathrm{nM})$ was added to $95 \mathrm{D}$ cells.

Despite recent advances in research, lung cancer remains a top killer in cancer-related deaths. In China, the 5-year survival rate of lung cancer patients is $<10 \%$. WHO warns that lung cancer and AIDS will be the two top causes of death in the 21 th century. Metastasis is found in $90 \%$ of lung cancercaused deaths. Since metastasis involves different cellular processes than those that participate in the onset and early stages of tumorigenesis, metastatic cancers can be more effectively treated if the metastasis process can be specifically targeted by therapeutics. Moreover, because the survival rate for patients diagnosed with metastatic lung cancer is dramatically lower than that of patients diagnosed with preor non-metastatic forms, identifying key molecules involved in metastasis shall greatly help early diagnosis of metastatic lung cancers, thus reducing patient fatality. Our results point to the direction that RhoGDI2 may function as a potential molecular marker in metastatic lung cancer, which warrants further investigation of its interaction with other signalling pathways involved in lung cancer.

\section{References}

1. Hirsch FR, Franklin WA, Gazdar AF and Bunn PA Jr: Early detection of lung cancer: Clinical perspectives of recent advances in biology and radiology. Clin Cancer Res 7: 5-22, 2001.

2. Stafford LJ, Vaidya KS and Welch DR: Metastasis suppressors genes in cancer. Int J Biochem Cell Biol 40: 874-891, 2008.

3. Gildea JJ, Seraj MJ, Oxford G, et al: RhoGDI2 is an invasion and metastasis suppressor gene in human cancer. Cancer Res 62: 6418-6423, 2002.

4. Scherle P, Behrens T and Staudt LM: Ly-GDI, a GDPdissociation inhibitor of the RhoA GTP-binding protein, is expressed preferentially in lymphocytes. Proc Natl Acad Sci USA 90: 7568-7572, 1993 .
5. Titus B, Frierson HF Jr, Conaway M, et al: Endothelin axis is a target of the lung metastasis suppressor gene RhoGDI2. Cancer Res 65: 7320-7327, 2005.

6. Theodorescu D, Sapinoso LM, Conaway MR, Oxford G, Hampton GM and Frierson HF Jr: Reduced expression of metastasis suppressor RhoGDI2 is associated with decreased survival for patients with bladder cancer. Clin Cancer Res 10: 3800-3806, 2004.

7. Ma L, Xu G, Sotnikova A, et al: Loss of expression of LyGDI (ARHGDIB), a rho GDP-dissociation inhibitor, in Hodgkin lymphoma. Br J Haematol 139: 217-223, 2007.

8. Cho HJ, Baek KE, Park SM, et al: RhoGDI2 expression is associated with tumor growth and malignant progression of gastric cancer. Clin Cancer Res 15: 2612-2619, 2009.

9. Tapper J, Kettunen E, El-Rifai W, Seppala M, Andersson LC and Knuutila S: Changes in gene expression during progression of ovarian carcinoma. Cancer Genet Cytogenet 128: 1-6, 2001.

10. Zhang Y and Zhang B: D4-GDI, a Rho GTPase regulator, promotes breast cancer cell invasiveness. Cancer Res 66: 5592-5598, 2006

11. Brighton PJ, Szekeres PG and Willars GB: Neuromedin U and its receptors: structure, function, and physiological roles. Pharmacol Rev 56: 231-248, 2004.

12. Nelson J, Bagnato A, Battistini B and Nisen P: The endothelin axis: Emerging role in cancer. Nat Rev Cancer 3: 110-116, 2003.

13. Moissoglu K, McRoberts KS, Meier JA, Theodorescu D and Schwartz MA: Rho GDP dissociation inhibitor 2 suppresses metastasis via unconventional regulation of RhoGTPases. Cancer Res 69: 2838-2844, 2009.

14. Wu Y, Moissoglu K, Wang H, et al: Src phosphorylation of RhoGDI2 regulates its metastasis suppressor function. Proc Natl Acad Sci USA 106: 5807-5812, 2009.

15. Cantley LC: The phosphoinositide 3-kinase pathway. Science 296: 1655-1657, 2002.

16. Manning BD and Cantley LC: Akt/pkb signaling: Navigating downstream. Cell 129: 1261-1274, 2007.

17. Shimizu M, Saitoh Y and Itoh H: Immunohistochemical staining of Ha-ras oncogene product in normal, benign, and malignant human pancreatic tissues. Hum Pathol 21: 607-612, 1990.

18. Zhang Y, Rivera Rosado LA, Moon SY and Zhang B: Silencing of D4-GDI inhibits growth and invasive behavior in MDA-MB231 cells by activation of Rac-dependent p38 and JNK signaling. J Biol Chem 284: 12956-12965, 2009.

19. Tang JM, He QY, Guo RX and Chang XJ: Phosphorylated Akt overexpression and loss of PTEN expression in non-small cell lung cancer confers poor prognosis. Lung Cancer 51: 181-191, 2006.

20. Chun KH, Kosmeder JW II, Sun S, et al: Effects of deguelin on the phosphatidylinositol 3-kinase/Akt pathway and apoptosis in premalignant human bronchial epithelial cells. J Natl Cancer Inst 95: 291-302, 2003

21. Sos ML, Fischer S, Ullrich R, et al: Identifying genotypedependent efficacy of single and combined PI3K- and MAPKpathway inhibition in cancer. Proc Natl Acad Sci USA 106: 18351-18356, 2009.

22. Sarbassov DD, Guertin DA, Ali SM and Sabatini DM: Phosphorylation and regulation of Akt/PKB by the rictor-mTOR complex. Science 307: 1098-1101, 2005.

23. O'Reilly KE, Rojo F, She QB, et al: mTOR inhibition induces upstream receptor tyrosine kinase signaling and activates Akt. Cancer Res 66: 1500-1508, 2006. 